\author{
„DIE WISSENSBASIERTE BIOÖKONOMIE GENERIERT MIT \\ BIOLOGISCHEN RESSOURCEN PRODUKTE, VERFAHREN \\ UND DIENSTLEISTUNGEN IN ALLEN WIRTSCHAFTLICHEN \\ SEKTOREN.“
}
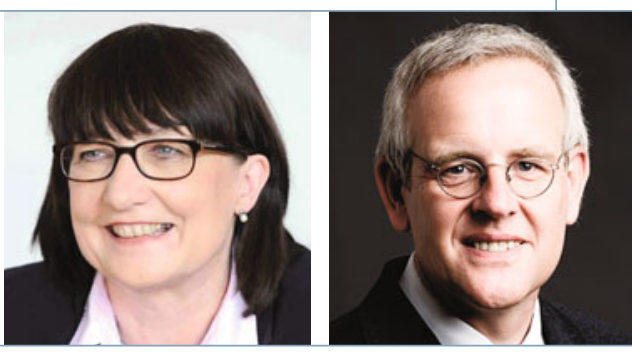

Christine Lang und Holger Zinke

\title{
Das biologische Wissen ist Treiber der Bioökonomie
}

Biotechnologie ist seit jeher ein spannendes und lohnendes Wirkungsfeld für Wissenschaft, Technologieunternehmen, Industrie und aber auch für Investoren und Finanzmarkt.

Es ist nicht schwer vorauszusagen, dass diese Gebiete in den kommenden Jahren neue effiziente Prozesse hervorbringen werden, die Klima und Umwelt schonen. Die biobasierte Wirtschaft wird auch völlig neue Produkte hervorbringen. Auf den Biotechnologietagen Mitte Mai in Stuttgart wurden etwa Dübel aus Bioplastik verteilt. Autohersteller wie Hyundai oder Konsumgüterriesen wie Procter \& Gamble und Coca Cola setzen verstärkt auf Bauteile und Verpackungen aus nachwachsenden Rohstoffen, die den Ausstoß klimaschädlicher Gase vermindern. Es ist es möglich, mithilfe von Mikroorganismen Seltene Erden zu gewinnen und Kohlendioxid als Rohstoff für Fermentation von Chemikalien zu nutzen. Eine besonders zahnschonende Zahnpasta mit Milchsäurebakterien oder Brustimplantate mit einer Beschichtung aus rekombinant hergestellter Spinnseide, die weniger AbstoBungsreaktionen hervorrufen, helfen Kosten im Gesundheitssystem zu sparen. Diese HightechVerfahren und -produkte sind im Wesentlichen wissensbasiert. Geistiges Eigentum erhält hier einen besonders hohen Hebel.

Die Beispiele zeigen: Die industrielle Biotechnologie ist innerhalb der Bioökonomie bereits heute ein bedeutendes Wirtschaftssegment, das im technologiestarken aber rohstoffarmen Deutschland eine besondere Bedeutung einnimmt.

Das Thema hat damit auch politische und wirtschaftsstrategische Bedeutung. Dies ist ein Fazit, das der Bioökonomierat in seinem aktuellen Eckpunktepapier zieht ${ }^{1}$.

Der Bioökonomierat, der als beratendes Gremium von der Bundesregierung berufen wird, und seit Herbst 2012 in einer zweiten Periode in neuer Zusammensetzung arbeitet ${ }^{1}$, sieht das biologische Wissen in der industriellen Anwendung der Biotechnologie in allen Farben und Facetten als zentralen Technologie- und Wirtschaftstreiber.

Bereits in der ersten Phase der Arbeit hat der Bioökonomierat eine Reihe von sektorspezifischen Berichten und Empfehlungen veröffentlicht ${ }^{2}$. Das zentrale Gutachten „Innovation Bioökonomie“"wurde 2010 von der Politik ange- nommen und etliche Punkte daraus in einer Nationalen Forschungsstrategie verankert. Eine wichtige Empfehlung war, Bioökonomie als „System“ zu betrachten und der Komplexität und Vernetzung der Akteure, aber auch der großen wettbewerblichen Herausforderung bei der Neudefinition von Wertschöpfungsketten und Rohstoffströmen Rechnung zu tragen. Dabei wurde der Blick nicht nur national ausgerichtet, sondern auch eine globale Verantwortung einbezogen.

Die wissensbasierte Bioökonomie wird auch im neuen Eckpunktepapier als zukunftsfähiges biobasiertes Wirtschaftssystem betrachtet, das unter Nutzung biologischer Ressourcen Produkte, Verfahren und Dienstleistungen in allen wirtschaftlichen Sektoren erzeugt und bereitstellt. Die wichtigsten Aufgaben, um das Potenzial der Bioökonomie zu nutzen, werden sein:

- Einen Dialog in Politik und Gesellschaft zu führen und Bioökonomie ins Gespräch zu bringen.

- Die Zukunftsfähigkeit und Wettbewerbsfähigkeit eines neuen Wirtschaftsbilds mit seinen Chancen (national und global) auszuloten.

- Forschung und Entwicklung sowohl grundlagen- als auch anwendungsorientiert $\mathrm{zu}$ intensivieren und über alle Wertschöpfungsketten und -netze zu fördern.

- Geeignete politische und wirtschaftliche Rahmenbedingungen zu schaffen, die zur Entwicklung der Bioökonomie beitragen.

- Aus- und Weiterbildung für qualifizierte Arbeitsplätze zu fördern, vor allem auf dem gleichzeitig hoch vernetzten sowie im Einzelnen sehr spezialisierten Gebiet der Bioökonomie.

Politischen Rückenwind gab es durch die im Jahr 2010 per Kabinettsbeschluss auf den Weg gebrachte „Nationale Forschungsstrategie BioÖkonomie 2030“. Wie auf den Biotechnologietagen zu hören war, wird durch eine koordinierte Aktivität verschiedener Ministerien eine gemeinsamen Politikstrategie formuliert. Dies unterstreicht weiter den positiven politischen Gestaltungswillen für eine kohärente Strategie zur Etablierung dieses Wirtschaftssystems.

Denn positiv-proaktives politisches Handeln zur Gestaltung der Rahmenbedingungen und der konsequenten Verfolgung strategischer Prio-

\footnotetext{
1 www.biooekonomierat.de

${ }^{2}$ www.whitehouse.gov/sites/default/files/microsites/ostp/national_bioeconomy_blueprint_april_2012.pdf
}

ritäten auf dem Weg zur biobasierten Wirtschaft wird benötigt. Mit einem ehrgeizigen „National Bioeconomy Blueprint“ hat die US-Regierung im April letzten Jahres vorgelegt ${ }^{2}$. Interessanterweise wird dort die „rote“ Biotechnologie ausdrücklich in die Betrachtungen eingeschlossen. Damit wird der pharmazeutisch-medizinische Bereich als bedeutender Wirtschaftssektor einer wissensbasierten Bioökonomie identifiziert, der in den vergangenen zwei Jahrzehnten massiv durch biologisches Wissen transformiert („biologisiert“) worden ist. Die US-amerikanische Volkswirtschaft hat in den vergangenen Jahrzehnten von dieser Entwicklung sehr profitiert, Deutschland als einstige „Apotheke der Welt“ weniger.

Nicht zuletzt aus dieser Erfahrung heraus ist der politische Wille begrüßenswert, zu einer kohärenten Strategie zu kommen - bei allen unterschiedlichen Sichtweisen, Schwerpunktsetzungen und zum Teil auch Widersprüchen und Konflikten aus Sicht der biologisch ausgerichteten Wissenschaft und Wirtschaft eine sehr begrüßenswerte Entwicklung.

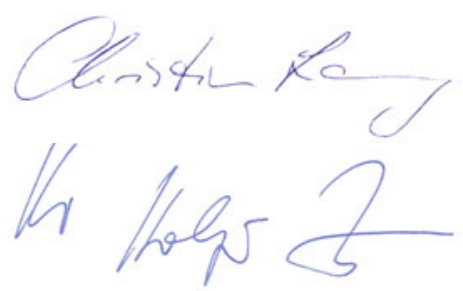

Christine Lang und Holger Zinke

Korrespondenzadressen:

Prof. Dr. Christine Lang

CEO, ORGANOBALANCE GmbH

Gustav-Meyer-Allee 25

D-13355 Berlin

Tel.: 030-46307200

Fax: 030-46307210

lang@organobalance.de

www.organobalance.com

Dr. Holger Zinke

CEO, B.R.A.I.N Aktiengesellschaft

Darmstädter Straße 34-36

D-64673 Zwingenberg

Tel.: 06251-9331-12

Fax: 06251-9331-11

hz@brain-biotech.de

www.brain-biotech.de 